\title{
Gravity currents: a personal perspective
}

\author{
By HERBERT E. HUPPERT \\ Institute of Theoretical Geophysics, Department of Applied Mathematics and Theoretical Physics, \\ University of Cambridge, Centre for Mathematical Sciences, Wilberforce Road, \\ Cambridge CB3 0WA, UK \\ heh1@esc.cam.ac.uk
}

(Received 9 September 2005 and in revised form 6 February 2006)

Gravity currents, driven by horizontal differences in buoyancy, play a central role in fluid mechanics, with numerous important natural and industrial applications. The first quantitative, fluid-mechanical study of gravity currents, by von Kármán in 1940, was carried out before the birth of this Journal; the next important theoretical contribution was in 1968 by Brooke Benjamin, and appeared in this Journal more than a decade after its birth. The present paper reviews some of the material that has built on this auspicious start. Part of the fun and satisfaction of being involved in this field is that its development has been based on both theoretical and experimental contributions, which have at times been motivated and supported by field observations and measurements.

\section{Introduction}

Gravity currents occur whenever fluid of one density flows primarily horizontally into a fluid of different density. (Predominantly vertical motion is described more naturally as plumes and involves different principles of fluid mechanics, Turner 1979.) There are numerous natural and industrial occurrences of gravity current motions. These include: the spreading of honey on toast; the propagation of a relatively cold (and generally slightly wet) sea breeze, as happens occasionally across Britain and so regularly on the East coast of Australia, across Canberra, and at Perth (the Fremantle doctor); the occasional outflow of relatively saline water from the Black Sea through the Bosporous into the Sea of Marmara; the intrusion of giant umbrella clouds into the atmosphere following a volcanic eruption; the destructive evolution of a snow avalanche; and the flow of molten glass across a horizontal surface to make sheet glass.

This paper reviews the conceptual foundations used to understand and evaluate the evolution of gravity currents. Some of the latest results in specialized areas are highlighted. After an introduction, the paper ranges through the principal effects due to: flows at low Reynolds numbers; compositional density differences; density differences due to dilute particulate matter; density differences due to concentrated particulate matter; and, finally, the motion of a granular medium (with very little influence of the interstitial fluid). In addition, some effects due to permeable boundaries, rotation and propagation into a porous medium or an ambient which is either stratified or uniformly flowing will be considered.

The first quantitative study of gravity currents was undertaken by von Kármán (1940), who was asked by the American military to evaluate under what wind conditions poisonous gas released would propagate forward to envelop the enemy, rather than backwards to cause havoc among the troops who released the gas. Using 
Bernoulli's theorem, von Kármán showed that the velocity, $u$, of the nose followed by a layer of depth $h$, of relatively heavy fluid with density excess $\Delta \rho$ over that of the atmosphere $\rho_{o}$, was given by

$$
u /\left(g^{\prime} h\right)^{1 / 2}=F r,
$$

where the reduced gravity $g^{\prime}=\Delta \rho g / \rho_{o}, g$ is the acceleration due to gravity and the Froude number, $F r$, was evaluated by von Kármán to be $\sqrt{2}$. This is the condition that has generally been applied at the nose of a current propagating into a very deep ambient at high Reynolds number $R e=u h / v$, where $v$ is the kinematic viscosity.

Such was the reputation of von Kármán that (1.1) and the paper in which it appeared quickly became celebrated, even though von Kármán had not considered the effects of wind in the atmosphere or pointed out that (1.1) is but one equation for the two unknowns $u$ and $h$. In 1968, Brooke Benjamin, who had just ceased being the co-editor (with George Batchelor) of this Journal, revisted the problem and argued that von Kármán had used Bernoulli's theorem incorrectly (by taking a contour through a turbulent region) and rederived (1.1) by the use of a momentum integral, or flow force as Benjamin called it. Benjamin expressed surprise that he obtained the same result as von Kármán, and was clearly somewhat distressed by this. However, given that the starting point of both scientists was an (admittedly different) integral of the Euler equations, there was not any possibility that they could have arrived at a different result. In addition, since $u, g^{\prime}$ and $h$ are the only variables in the problem, the non-dimensional quantity $u /\left(g^{\prime} h\right)^{1 / 2}$ has to be a constant. If the depth of the ambient, $H$, is comparable to $h$ then the Froude number becomes a function of $h / H$ (Huppert \& Simpson 1980).

Expressions in the form of (1.1) have been used in (almost) all studies of gravity currents propagating at high Reynolds number as a form of conservation of momentum and have sometimes been expressed as indicating that, at high Reynolds number, a gravity current is controlled at the nose. Gravity currents propagating at low Reynolds number behave quite differently, and, indeed, are not generally controlled, or even influenced, at the nose at all.

The next section briefly reviews the approach to studying low-Reynolds-number gravity currents, before continuing the above development for high-Reynolds-number currents. The material is biased towards areas I have been involved with and know best. I hope this stance is understandable, though it means that a number of important contributions have not been mentioned. An alternative, interesting view on the fundaments and applications of gravity current flows is contained in Simpson (1997).

\section{Viscous gravity currents}

Viscous gravity currents propagate under a balance between viscous and buoyancy forces (and the two fluids are usually considered essentially immiscible). In all problems so far solved the viscous fluid has been assumed to spread as a thin layer and the concept of lubrication theory has been appropriate. Thus the velocity profile is parabolic and conservation of mass leads to a governing nonlinear partial differential equation in space and time for the unknown free-surface height $h(x, t)$ as depicted in figure 1 $(a)$. Thus, for example, for a current spreading along a horizontal base in either a wide channel or an axisymmetric situation, the governing equation is 

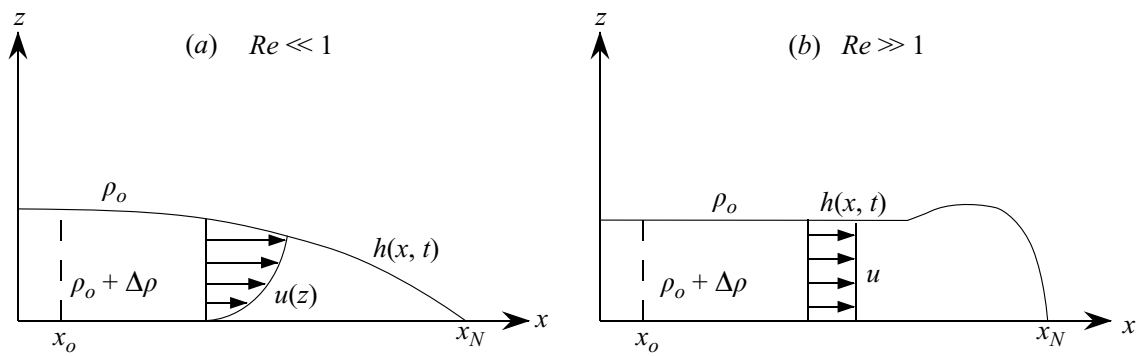

FIGURE 1. A sketch of a propagating gravity current showing the notation and (a) a parabolic velocity profile for $R e \ll 1$ and $(b)$ the uniform velocity profile for $R e \gg 1$.

(Barenblatt 1978; Huppert 1982a)

$$
\frac{\partial h}{\partial t}-\frac{\beta}{x^{n}} \frac{\partial}{\partial x}\left(x^{n} h^{3} \frac{\partial h}{\partial x}\right)=0,
$$

where $\beta=\frac{1}{3} g / \nu, n=0$ in Cartesian coordinates and $n=1$ in radial coordinates (where $x$ is interpreted as the radius). Equation (2.1) is valid under the assumption that the Bond number $B=\rho g l^{2} / T \gg 1$, where $T$ is the surface tension and $l$ the horizontal extent of the current, so that surface tension effects along the interface and nose can be neglected. To (2.1) must be added an overall volume conservation relationship, which is generally taken of the form

$$
\int_{o}^{x_{N}(t)}(2 \pi x)^{n} h(x, t) \mathrm{d} x=q t^{\alpha},
$$

where $x_{N}(t)$ is the extent of the current and the volume is assumed to be given by $q t^{\alpha}$, where, for example, $\alpha=0$ represents a constant-volume current and $\alpha=1$ represents a current fed by a constant flux.

A similarity form of solution is easily obtained for (2.1) and (2.2) and is written as (Huppert 1982a, 1986)

$$
h(\boldsymbol{x}, t)=\zeta_{N}^{2 / 3}\left(q^{2-n} / \beta\right)^{1 /(5-n)} t^{[(2-n) \alpha-1] /(5-n)} \Phi\left(\zeta / \zeta_{N}\right),
$$

where the similarity variable

$$
\zeta=\left(\beta q^{3}\right)^{-1 /(5+3 n)} x t^{-(3 \alpha+1) /(5+3 n)}
$$

and $\zeta_{N}(\alpha, n)$ is determined by (2.2). The similarity variable (2.4) indicates immediately, with no further analysis, that the extent of the current is given by

$$
x_{N}=\zeta_{N}\left(\beta q^{3}\right)^{1 /(5+3 n)} t^{(3 \alpha+1) /(5+3 n)} \text {. }
$$

Experimental results for the rate of propagation of such viscous spreading are in excellent agreement with the theoretical determination (Huppert 1982a, 1986, 2000). The motivation for the original study was the availability of wonderful data on the spreading of the lava dome formed in the crater of the Soufrière of St Vincent in the West Indies after the eruption of 1979. The resultant lava dome slowly increased in volume, and spread horizontally for five months across the crater floor, by which time the pancake-shaped dome had a height of $130 \mathrm{~m}$, a mean diameter of $870 \mathrm{~m}$ and a volume of $5 \times 10^{7} \mathrm{~m}^{3}$ (Huppert et al. 1982). Observations of the volume then indicated that $\alpha=0.66$, which should lead to a radial time dependence, from (2.5), of $(3 \alpha+1) / 8=0.37$, in good agreement with the measured value of 0.39 . In 


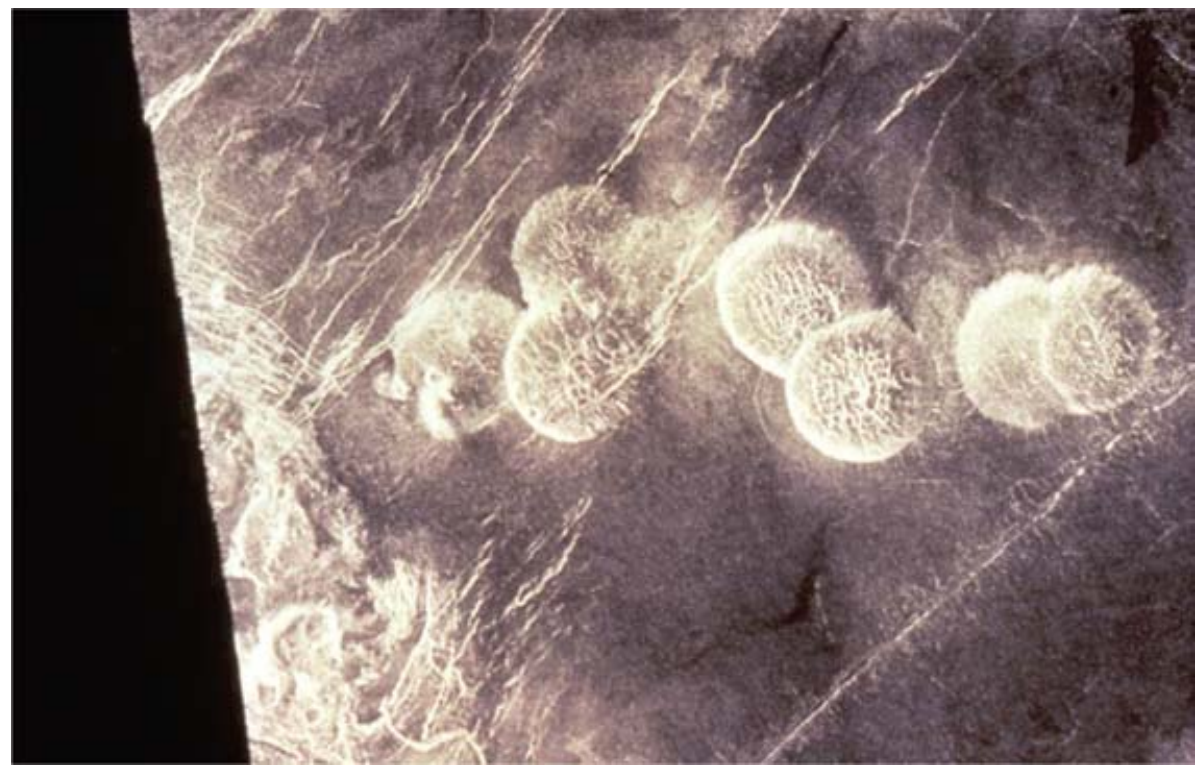

FIGURE 2. A series of lava domes on Venus.

addition, (2.5) can then be inverted to determine a value for the kinematic viscosity of $6 \times 10^{7} \mathrm{~m}^{2} \mathrm{~s}^{-1}$. Considerable further work has been done to make this initial model more realistic by including the effects of compressibility of the interstitial gas in the magma (Jaupart 1991), solidification of the thin upper carapace of the lava dome (Fink \& Griffiths 1990, 1998; Griffiths 2000) and non-Newtonian rheology of the spreading fluid (Balmforth, Burbidge \& Craster 2001; Balmforth, Craster \& Sassi 2004). The theoretically determined shape, (2.3), has also been successfully used to describe the shape of the numerous lava domes recently observed on Venus (McKenzie et al. 1992), a photograph of which is shown in figure 2.

The flow at low Reynolds number of a viscous fluid down a slope occurs in many different situations: the splattered wet mud down a car windscreen; liquid detergent on a slanted plate; and rainwater down the roof of a glass conservatory. A fundamental problem in this area, which has acted as the foundation of many further studies, is the question of whether a broad band of viscous fluid, uniform in depth across the slope, can continue to flow down the slope in a fashion independent of the cross-slope coordinate. This situation was first considered by Huppert (1982b) who showed, again under the assumption that $B \gg 1$, that there is a parabolic velocity profile and that the free surface $h(x, t)$ is governed by

$$
\frac{\partial h}{\partial t}+\left(g \sin \theta^{\prime} / v\right) h^{2} \frac{\partial h}{\partial x}=0
$$

where $x$ is the coordinate down the slope, which is inclined at angle $\theta$ to the horizontal. By use of either the theory of characteristics or by similarity theory, Huppert showed that the appropriate theoretical solution of this two-dimensional flow is

$$
h=(v / g \sin \theta)^{1 / 2} x^{1 / 2} t^{-1 / 2}
$$

for

$$
0 \leqslant x<x_{N}=\left(9 A^{2} g \sin \theta / 4 v\right)^{1 / 3} t^{1 / 3}
$$


where $A$ is the initial cross-sectional area of the current. The relationship (2.7a) indicates that at any time the free surface increases in thickness down the slope like the square root of the coordinate down the slope until $x=x_{N}$ at which point the current ends (abruptly in this solution that neglects surface tension) and that at any point down the slope, once the current has passed overhead, its thickness decreases like the square root of time.

To test the validity of this theoretical prediction, Huppert conducted experiments with different viscous fluids flowing down slopes of different angles to the horizontal, an example of which makes up movie $1 . \dagger$ Initially the motion was virtually independent of the cross-slope coordinate and in good agreement with the predictions of (2.7). However, after some time the flow front seemed spontaneously to develop a series of small-amplitude waves of fairly constant wavelength across the slope. The amplitude of the waves increased in time as the maxima (points furthest down the slope) travelled faster than the minima. The wavelength remained unaltered. For silicon oils the subsequent shape was a periodic, triangular front with tightly rounded maxima, connected, by very straight portions at an angle to the slope, to extremely pointed minima. For glycerine, the shape was also periodic, though with much less tightly rounded maxima, again connected by extremely straight portions, almost directly down the slope, to very broad minima. To my knowledge no other shape has been seen for different fluids. The initial instability must be due to the effects of surface tension, which were neglected in the two-dimensional analysis. Incorporating these effects at the tip, Huppert was able to show that the wavelength of the instability was represented well by

$$
\lambda=\left(7.5 A^{1 / 2} T / \rho g \sin \theta\right)^{1 / 3},
$$

independent of the coefficient of viscosity, which only sets the time scale of the instability and its onset.

\section{Compositional-driven currents}

In (almost) all situations a current whose density difference is due to a dissolved component of negligible diffusivity, such as salt, propagates in such a way that its horizontal extent is generally very much larger than its vertical extent. A natural example of such a current is shown in figure 3 and laboratory examples make up movie 2. Under the assumption that the Reynolds number is large, the balance is then between inertial and buoyancy forces and standard shallow-water theory (Whitham 1974) can be applied. Alternatively, Huppert \& Simpson (1980) introduced the concept of a 'box model', which considers the simple model that results from assuming the current to evolve through a series of equal-area rectangles, or equal-volume cylinders, as appropriate, with no variations of any properties in the horizontal. (An integral justification of this approach is given in the Appendix of Harris, Hogg \& Huppert 2001). With the assumption that the ambient is sufficiently deep that the Froude number is constant, this approach leads to the relationship

$$
x_{N}=C(F r)\left(g^{\prime} A\right)^{(2-n) / 6} t^{(4-n) / 6},
$$

where $C(F r)$ is dependent only on the Froude number and $A$ is the cross-sectional area in two dimensions $(n=0)$ and the volume in three dimensions $(n=1)$ of the

$\dagger$ The movies referred to are available as a supplement to the online version of the paper. 


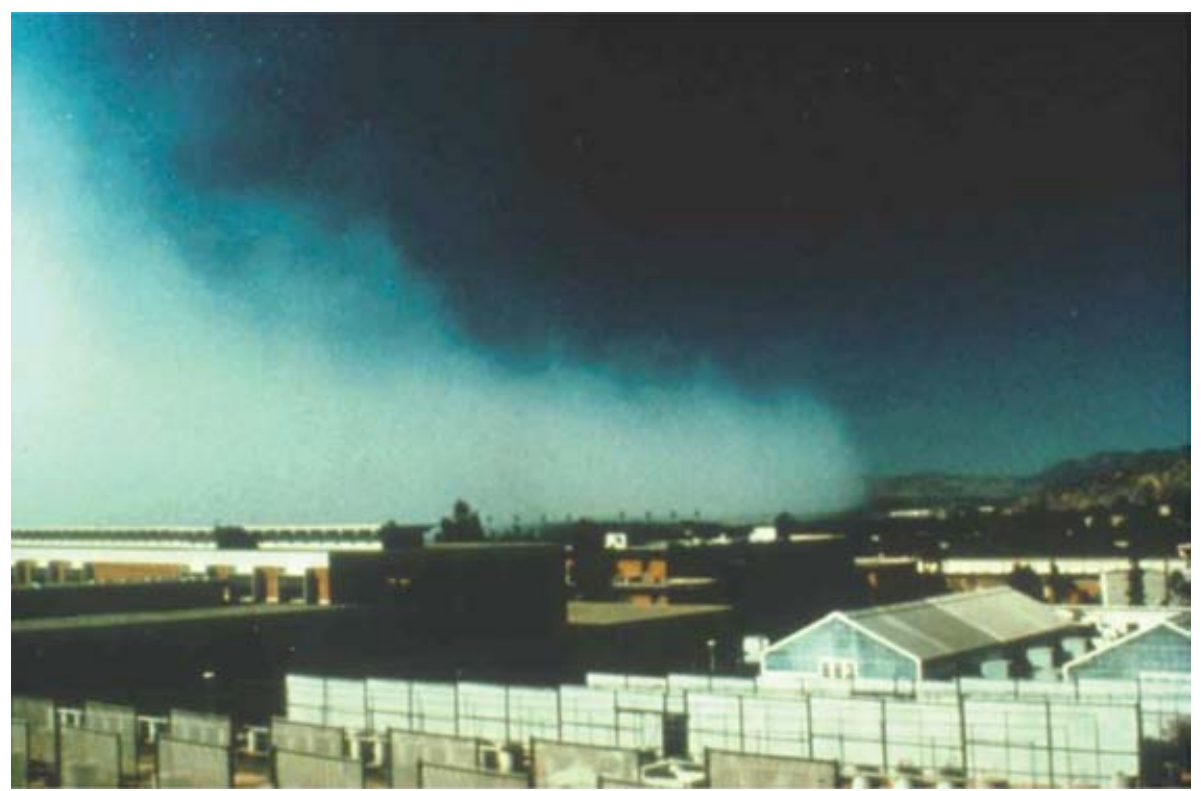

FIGURE 3. A large-Reynolds-number compositional gravity current over the Los Angeles Basin. Almost every morning the sun shines down on Southern California and the neighbouring Pacific Ocean. The atmosphere above the land surface becomes relatively warm and less dense than that above the sea. The horizontal density difference results in a gravity current of relatively cold, moist air, which spreads across the California countryside (photographer: G. R. Stevens).

current. Note that $g^{\prime}$ and $A$ only appear as the product $g^{\prime} A$, which is conserved even if mixing between the two fluids occurs.

The use of similarity theory applied to the shallow-water equations (Hoult 1972; Chen 1980; Bonnecaze, Huppert \& Lister 1993; Bonnecaze et al. 1995) leads to a relationship which differs only in the pre-multiplicative constant in (3.1), and then only by a relatively small quantitative amount. (But that is the power of the correct dimensional constraints!) However, before a current released from behind a lock enters the self-similar propagation phase described by (3.1), it goes through a rather different phase, described as 'slumping'. Both experiments and shallow-water models indicate that for between 3 and 10 lock-lengths, which increases as the ratio $h / H$ decreases, the velocity of propagation in the slumping phase is constant (Huppert \& Simpson 1980; Rottman \& Simpson 1983).

Entrainment of ambient fluid into the flow has been investigated both theoretically and experimentally (Hallworth et al. 1993, 1996) by following the intrusion of an alkaline current into an acidic ambient. Entrainment takes place almost entirely at the head of the current owing to shear instabilities on the interface between the current and the ambient and by the over-riding of the (relatively less dense) ambient fluid as the head propagates over it. An entrainment or dilution ratio $E$, defined as the ratio of the volumes of ambient and original fluid in the head, which hence must be non-negative, can be shown by dimensional analysis, and was confirmed by experiment, to be independent of $g^{\prime}$, and to be given for a gravity current in a channel by $E=\left[1-c_{1} y_{N} / A_{s}^{1 / 2}\right]^{-c_{2}}-1$, where $A_{s}$ is the cross-sectional area of fluid in the head at the end of the slumping phase, $y_{N}$ is the position of head beyond the slumping 
point, and $c_{1} \approx 0.05$ and $c_{2} \approx 1.5$ are empirical constants determined by the roughness of the floor.

In an independent study, Hacker, Linden \& Dalziel (1996) conducted three experiments during which they measured the spatial and temporal variations of the intensity of light leaving the tank from a uniformly adjusted background lighting after it had been attenuated by absorption by the dye through which it passed. From the intensity they back calculate the concentration of dye and hence the amount of mixing. These calculations indicate a complex internal density structure and suggest that substantial mixing occurs in the early stages of the flow. This is in contrast to the detailed results of Hallworth et al. $(1993,1996)$ from many experiments, who observe that there is comparatively little entrainment into the head that emerges at the end of the slumping phase. A resolution of these different conclusions is awaited.

As the current from the instantaneous release of a constant volume flows either along a two-dimensional channel or away from the origin in an axisymmetric situation, the spreading makes the depth decrease, and thus, by the Froude number condition at the front (1.1), the velocity of the front decreases also. What happens, however, if the fluid is released from an outer radius and travels inward in either a sector or the full axisymmetric case? Examples where this might occur in nature include a bore entering a narrowing channel and a turbidity current generated along the edge of a lake. Because the fluid spreads horizontally as it propagates, the height at the nose should decrease. However, because of the geometrical constraints, as the fluid approaches the centre the height should increase. Which effect dominates? Somewhat to my surprise, both happen - but sequentially. In a series of experiments, backed up by numerical integration of the shallow-water equations, Hallworth, Huppert \& Ungarish (2003) showed that the current first decreases in thickness and slows down, until it is about halfway between the release point and the centre; thereafter the thickness increases, as does the velocity. This can be rather simply understood mathematically from just the axisymmetric continuity equation

$$
\frac{\partial h}{\partial t}+\frac{\partial(u h)}{\partial r}+\frac{u h}{r}=0,
$$

where $r$ is the radial coordinate measured from the centre. In a balance of terms usually $\partial / \partial r \sim 1 / r$. However in this case, the rate of change with radius, $\partial / \partial r$, represents the change with the inward radial scale, and therefore $\sim 1 /\left(R_{o}-r\right)$, where $R_{o}$ is the (outer) radius of release. Incorporating this into (3.2) leads to

$$
\frac{\partial h}{\partial t} \sim u h\left(\frac{1}{R_{o}-r}-\frac{1}{r}\right),
$$

which changes sign at $r=R_{o} / 2$ and therefore indicates that the turnover point is about halfway. An accurate similarity solution of the second kind (Barenblatt 1996) has recently been obtained by Slim \& Huppert (2004) which examines, via a phase-plane analysis, the motion just as the current reaches the centre.

\section{Particulate-laden currents}

When heavy (or possibly relatively less dense) particles drive the flow the major new addition to the advective effects is that the particles fall (or rise) out of the flow and the driving buoyancy continually decreases. Examples of this form of motion are the terrifying flows seen in lower Manhattan on September 11, 2001 and the awesome hot ground-hugging pyroclastic flows which can be the life-threatening destructive 


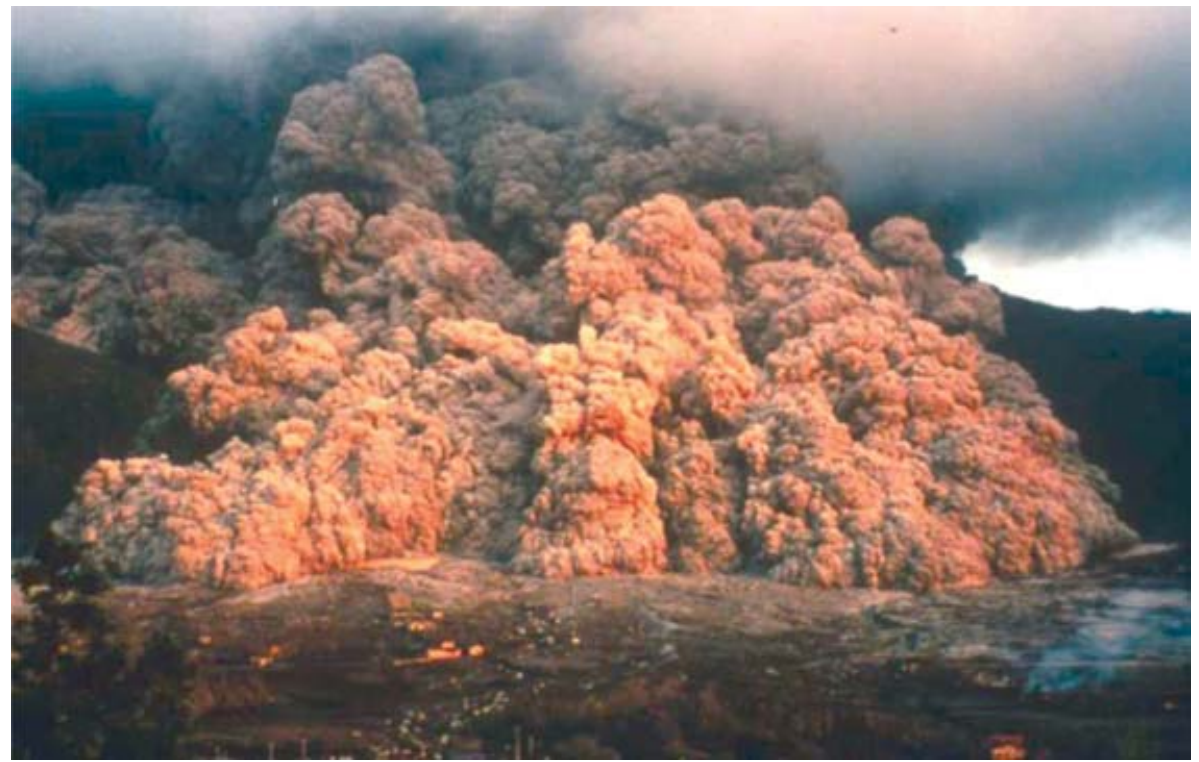

FIgURE 4. A hot, particle-laden gravity current, or pyroclastic flow, from the eruption of Mt. Unzen in Japan.

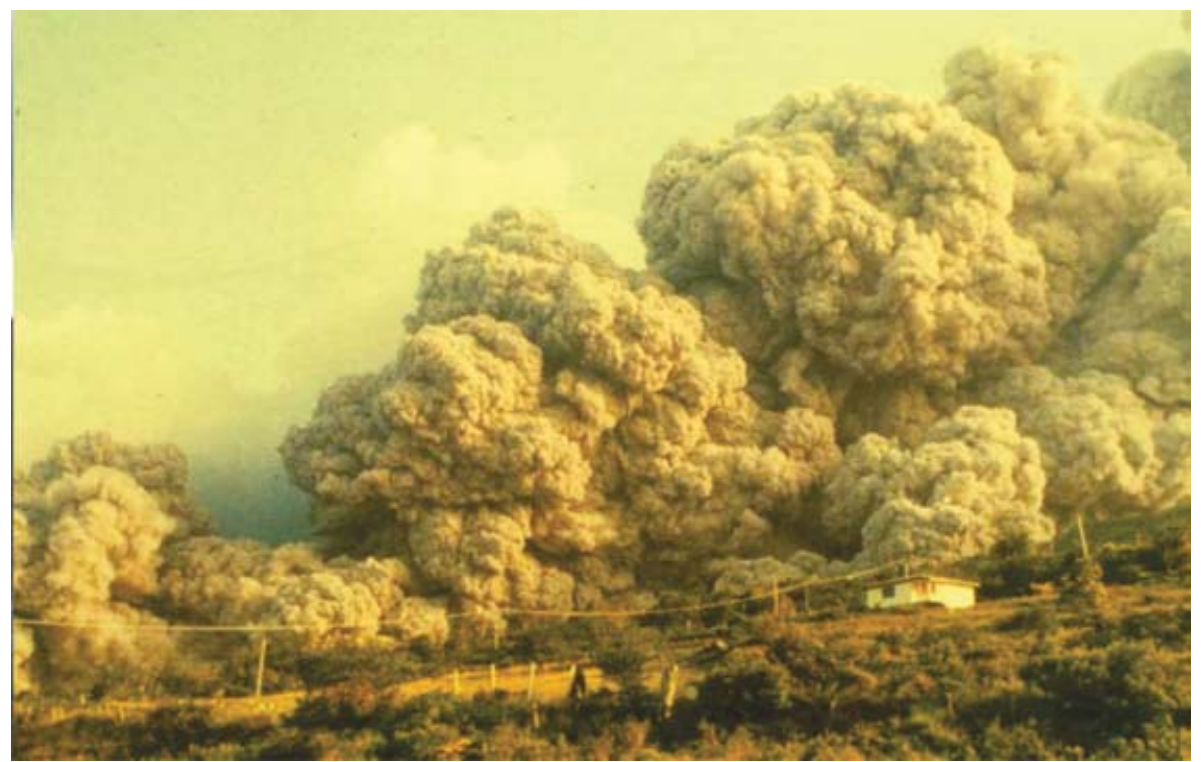

Figure 5. A pyroclastic flow on Montserrat in the Caribbean (photographer: R. S. J. Sparks).

result of volcanic eruptions such as occurred recently at Pinatubo in Indonesia, Unzen in Japan (figure 4) and Montserrat in the Caribbean (figure 5). After a sufficient mass of particles has dropped to the ground, so that the bulk density of this hot flow is no longer greater than that of the surrounding atmosphere, the current can suddenly rise quite dramatically, taking much of the remaining particulate matter high into the atmosphere. Laboratory simulations of such flows are shown in movies $2(c-e)$. 
The approach most frequently taken to analyse the sedimentation if the concentration is not too large is to assume that the (high-Reynolds-number) flow is sufficiently turbulent to maintain a vertically uniform particle concentration in the main body of the current. However, at the base of the flow, where the fluid velocities diminish appreciably, the settling of particles occurs at the (low-Reynolds-number) Stokes velocity $V_{s}$ in otherwise quiescent fluid. Quantitatively, this indicates that, neglecting particle advection for the moment and assuming that the particles are all of one size, if $N_{p}$ (which is possibly a function of time and position) denotes the total number of particles per unit horizontal area in a layer of depth $h$, the change of $N_{p}$ in time $\delta t, \delta N_{p}$, due only to the sedimentation, is given by $\delta N_{p}=-V_{s} C_{0} \delta t$, where $C_{0}$ is the (number) concentration (per unit volume) just above the base of the flow. Vigorous turbulent mixing implies that $C_{0}=N_{p} / h$, which (on taking the appropriate infinitesimal limits) indicates that $\mathrm{d} N_{p} / \mathrm{d} t=-V_{s} N_{p} / h$, a relationship which has been verified by experiments in which the particles were suspended in a thermally convecting fluid (Martin \& Nokes 1988) and used very successfully in many investigations since then. Incorporation of advection of particles by the mean flow then results in

$$
\frac{\mathrm{D}}{\mathrm{D} t} \phi \equiv \frac{\partial \phi}{\partial t}+\boldsymbol{u} \cdot \nabla \phi=-V_{s} \phi / h,
$$

where $\phi$ is the volume concentration of particles.

Shallow-water equations incorporating (4.1) are easily derived (Bonnecaze et al. 1993, 1995; Huppert 1998). There are no similarity solutions and recourse, in general, has to be made to numerical solution. There is very good agreeent between the numerical solutions and experimental determinations carried out specially to test them.

Aside from numerical solutions, it is also possible to develop asymptotic analytic solutions based on the smallness of $\beta_{S}=V_{S} /\left(g_{o}^{\prime} h_{o}\right)^{1 / 2}$, where $g_{o}^{\prime}$ is the initial reduced gravity of the system (Harris et al. 2001). This is an example of the interesting problem which can be stated in general as the nonlinear partial differential equation

$$
\mathscr{N}_{1}[\Phi(x, t)]=\varepsilon \mathscr{N}_{2}[\Phi(x, t)],
$$

where $\mathscr{N}_{1}$ and $\mathscr{N}_{2}$ are nonlinear operators in some spatial coordinate $x$ and time $t$. For $\varepsilon \equiv 0$ there is a similarity solution to (4.2) and a special value of $x, x_{*}$ say, such as that at the nose of the current, which increases continually with time, of the form $x_{*}=f(t)$. However, for $\varepsilon \neq 0$, no matter how small, (4.2) does not have a similarity solution and eventually, for sufficiently large $t, x_{*}$ attains a constant. Thus, in the style of singular perturbation problems, for sufficiently large $t$ the solution for $\varepsilon=0$ departs from solutions for $\varepsilon \ll 1$. It must, on the other hand, be possible to obtain asymptotic solutions to (4.2) in a perturbative sense. Such a technique is constructed, in part, in Harris et al. (2001).

When there is an external flow present, as originally put to von Kármán, the propagation of the current is significantly influenced, and in a different way if it is propagating with the ambient flow or against it. Hallworth, Hogg \& Huppert (1998) and Hogg, Huppert \& Hallworth (2005) initiated an analysis and a series of laboratory experiments to investigate this case. In their first paper, they showed that for an instantaneous release of a fixed volume of dense fluid into a channel, the flow was dependent on the single non-dimensional parameter $\Lambda=U A /\left(l_{\infty}^{2} V_{S}\right)$, where $U$ is the effective mean external flow experienced by the current, evaluated to be 0.6 times the actual current (in agreement with an earlier evaluation by Simpson \& Britter 1980), $A$ is the cross-sectional area (or volume per unit width) of the fluid instantaneously released at the base of the flow, the reduced gravity of the particles of density $\rho_{P}$ is 


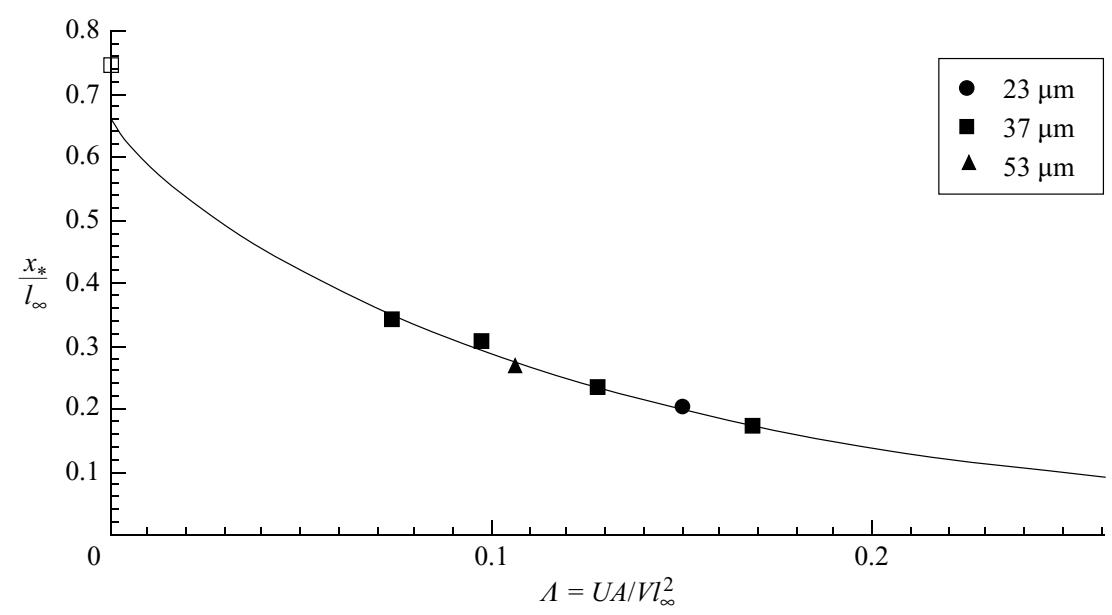

FIGURE 6. The theoretical prediction and experimental data of the maximum non-dimensional upstream penetration of a particulate gravity current in a uniform flowing ambient, $x_{*} / l_{\infty}$, as a function of $\Lambda=U A /\left(V_{S} l_{\infty}^{2}\right)$ for three different particle sizes.

given by $g_{P}^{\prime}=\left(\rho_{P}-\rho_{a}\right) g / \rho_{a}, \rho_{a}$ is the density of the ambient and $l_{\infty}=\left(g_{P}^{\prime} \phi_{o} A^{3} / V_{s}^{2}\right)^{1 / 5}$. The parameter $\Lambda$ represents the ratio of the mean external velocity to the settling velocity of the particles. When $\Lambda$ is small (little effect of the flow in the ambient), the flow is roughly the same upstream or downstream. As $\Lambda$ increases the effects of the flow in the ambient increase and the upstream flow is considerably restricted. However, even for large $\Lambda$ there will still be some upstream propagation - much to the disbelief and then dismay of the government official who asked me to carry out this work in order to show that a new dredging technique, in which mud suspended from the bottom of the harbour by jetting high-velocity water at it, would be swept out to sea by the outgoing tidal flow and not propagate at all upstream across the harbour and contaminate the highly valuable stocks of fish and oysters there! Figure 6 presents the theoretically determined maximum non-dimensional upstream distance of a particulate intrusion in a uniformly flowing ambient as a function of $\Lambda$ and the laboratory experimental data obtained using various size particles. The agreement shown is excellent; and one of the best I have ever obtained in my career, and hence my pleasure with it.

Extensions of this idea to an axisymmetric (rather than line) source and to a constant input flux (rather than an instaneous release from a point source) are summarized in Hogg \& Huppert (2001a,b) and Hogg et al. (2005) respectively, where the relevant parameters and shape of the current are carefully discussed.

An alternative approach is to develop full-scale CFD codes, or Navier-Stokes solvers, to follow particle-driven flows. This has been done with considerable success by Ungarish (Hallworth, Huppert \& Ungarish 2001 and Ungarish \& Huppert 2004) who compared the results with experimental data and the predictions of a one-layer shallow-water model. Numerical codes for this problem have also been described in Dalziel \& Linden (1997) and Härtel, Meiburg \& Necker (2000).

\section{Additional effects}

The preceding four sections have detailed some of the fundamental relationships for the propagation and evolution of a gravity current. Many other special effects are 
possible. We will describe some of these under the headings of: flows over permeable boundaries; flows in porous media; flows into a stratified ambient; and effects of rotation.

\subsection{Flows over permeable boundaries}

There are many natural and industrial situations where a gravity current flows over a permeable boundary and seeps into it. Examples include the protection of liquidcontaining structures by surrounding them with a deep gravel bed, the seepage of tidal inflow up a beach and the everyday occurrence of honey seeping into toast. The most important new ingredient is that fluid from the current continuously seeps through the boundary, which reduces the volume of the current and its horizontal propagation must eventually cease.

The flow of the current at low Reynolds number was considered by Acton, Huppert \& Worster (2001). The parabolic velocity profile in the current is augmented by a sink term in the local continuity equation which leads in two dimensions, the only situation that has been investigated, to

$$
\frac{\partial h}{\partial t}-\beta \frac{\partial}{\partial x}\left(h^{3} \frac{\partial h}{\partial x}\right)=-(k g / v)(1+h / l),
$$

which takes the place of (2.1). The term on the right-hand side represents the Darcy flow driven by a vertically linear pressure gradient into the porous medium below, which is categorized by the intrinsic permeability of the medium $k$, and $l$ denotes the length of the current. In contrast to the situation for an impermeable boundary, (5.1) has a similarity solution only for the special case of $\alpha=3\left(V \propto t^{3}\right)$, for which the flux into the current at the origin just balances that out of the current through the permeable boundary. For other values of $\alpha$ solutions of (5.1) must be obtained by numerical integration. This was done by Acton et al. (2001), who also carried out experiments to compare observational data on the length of the current as a function of time with their numerical solution. The result, as shown in figure 7, was an extremely good comparison, with a final run-out length which scales with $S_{H}=\left(A^{2} k\right)^{1 / 3}$ for $\alpha=0$, where $A$ is the cross-sectional area, or volume per unit width. For $\alpha<3$ all currents eventually stop; for $\alpha \geqslant 3$ all currents continue to propagate because the incoming flux overpowers the seepage. Note the similarity in concept between the slow loss of driving force due to seepage, considered here, and the loss of buoyancy due to slow particle fallout, considered in $\S 4$. The general approach discussed there, as outlined in Harris, Hogg \& Huppert (2001, 2002), has not been applied to this area, though no doubt it could be done so fruitfully.

When the current propagates at high Reynolds number the balance of forces is of course different, but the importance of seepage through the boundary is still paramount. This problem was initially considered by Thomas, Marino \& Linden (1998) and then extended by Ungarish \& Huppert (2000). The latter solved the shallow-water equations for a two-dimensional geometry numerically as well as obtaining analytical box-model solutions in both rectangular and axisymmetric geometries. This allowed closed-form solutions for the extent of the current as a function of time and its final extent to be determined, which were in fair agreement with both the two-dimensional experiments carried out by Thomas, Marino \& Linden (1998, 2004) and the numerical solutions. 


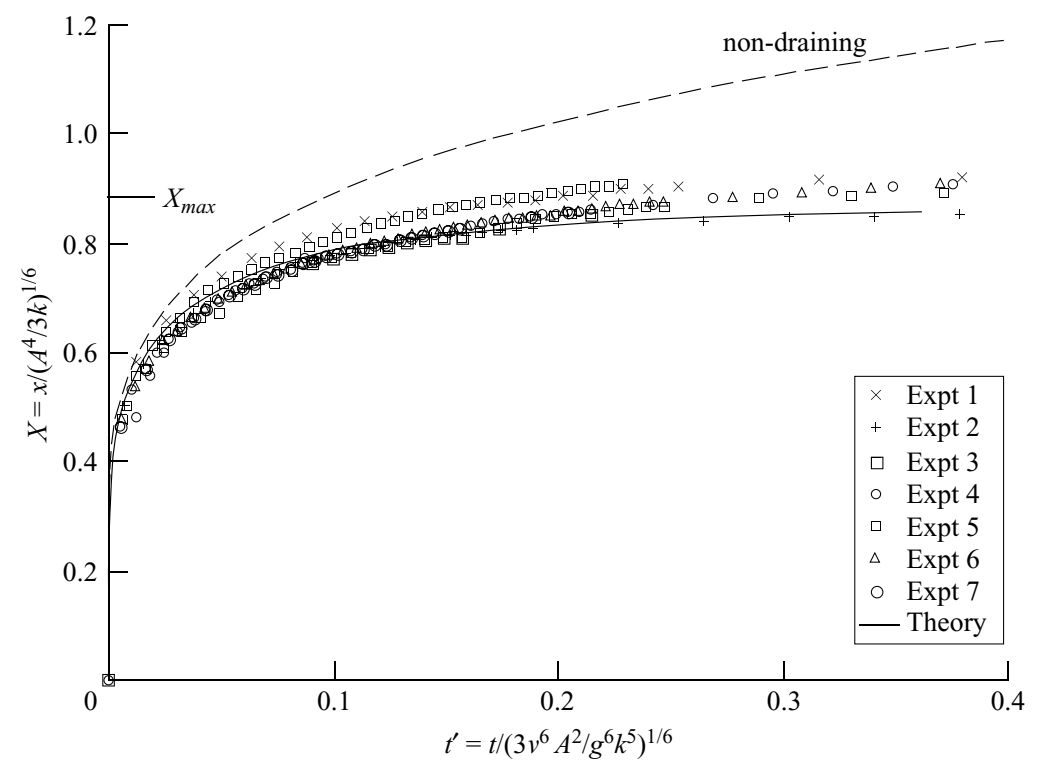

FIGURE 7. The non-dimensional length of a low-Reynolds-number gravity current flowing over a permeable boundary as a function of the dimensionless time for seven different experiments. The solid curve is the theoretical prediction determined from numerical integration of (5.1) and the dashed line is the similarity solution for an impermeable boundary given by (2.7).

\subsection{Flows in porous media}

The intrusion of a gravity current into a saturated porous medium has considerable similarities to the propagation of viscous gravity currents as described in $\S 2$. The twodimensional flow over an impermeable boundary has a similarity form of solution to the governing nonlinear advection-diffusion equation. For the instantaneous release of a finite volume of fluid the current spreads like $t^{1 / 3}$ and has a parabolic thickness distribution. This rate of spread and functional form of the height of the current was confirmed by experiments in Hele-Shaw cells, which yielded good agreement with the predictions, even down to the pre-multiplicative constants (Huppert \& Woods 1995). They also developed the theory and forms of solutions for a two-dimensional current propagating down an impermeable incline.

The axisymmetric situation has recently been investigated by Lyle et al. (2005). Their investigation was motivated by the problem of carbon dioxide sequestration. At the moment approximately 23 Gigatonnes of carbon dioxide are anthropogenically produced each year and the emission of such large amounts of carbon dioxide, a greenhouse gas, could drastically change aspects of the radiation balance in the atmosphere and threaten global climate. One way to ameliorate this problem is to store carbon dioxide, in a liquid state, in gigantic porous reservoir rock at considerable depth beneath the Earth's surface. This is currently being done at a number of sites around the world, most notably since 1996 at the Sleipner gas field in the North Sea. Lyle et al. (2005) showed that the axisymmetric spreading as a result of a constant input flux has a radius proportional to $t^{1 / 2}$ and carried out experiments to verify this conclusion. We are currently considering extra effects due to sloping and/or impermeable boundaries (Vella \& Huppert 2006) as well as applying the detailed results to interpreting the geological observations (Bickle et al. 2006). 
A study which combines effects described in this and the last subsection considers the flow of a gravity current in a porous medium above a boundary of much lower permeability (Pritchard, Woods \& Hogg 2001). They determine that, as before, the slow drainage through the slightly permeable lower boundary restricts the current to a finite length. This set-up is relevant to the seepage of oil or gas through layered media.

\subsection{Propagation under the base of a stratified ambient}

In all the foregoing discussion, it has been assumed that the ambient is homogeneous and not influenced by the intruding current (except for the two-layer calculations of Bonnecaze et al. 1993; Hallworth et al. 1998; Hogg et al. 2005). However, in both the atmosphere and the oceans the ambient may be stratified; and it immediately raises the question of why the solutions so far obtained are applicable in these more complicated, natural situations - or has something important been left out? What clearly has been left out of the homogeneous models is the possibility of exciting internal waves in the ambient by the propagation of the current, as seen clearly in movie 3. Viewed from another angle, the question that should be considered is what fraction of the potential energy stored at the outset in the intruding fluid is lost to internal wave motions, in contrast to that converted to the kinetic energy of the current and that which is dissipated. The first investigation of the influence of a stratified ambient considered the propagation of a saline current below a linearly stratified saline ambient in a rectangular container (Maxworthy et al. 2002). Their study was a combination of laboratory and numerical experiments. The numerical solutions, obtained from a full Boussinesq formulation, were in very good agreement with their measurements. They focused attention on the speed of propagation of the nose during only the initial stage of release from behind a lock, for which a good agreement between theory and numerical computation was obtained. Ungarish \& Huppert (2002) then extended this investigation by determining appropriate analytical solutions. Using the methods of characteristics on the nonlinear shallow-water equations, which neglects the influence of flow and waves in the ambient, they were able to obtain solutions for the front velocity as a function of the parameter $S$, defined as the ratio of the density difference between the fluid that makes up the bottom of the ambient and that at the top to the density difference between the fluid of the incoming current and that at the top. The results of their calculations, compared with the experimental results of Maxworthy et al. (2002), are shown in figure 8. This indicates that wave motion in the stratified ambient plays a relatively small role. Confirmation of this conclusion, based on evaluating the evolution of potential and kinetic energies in both the current and the ambient as functions of time, is presented in Ungarish \& Huppert (2006). A study of an intrusive gravity current into the interior of a stratified medium is presented in Ungarish (2005).

\subsection{Some effects of rotation}

Rotational effects, which can be dominant, for example, in rapidly rotating systems in the laboratory, in industrial machines or due to the rotation of the Earth, bring in the initially counter-intuitive effect that fluid flows at right angles to the pressure gradient, in analogy with some of my more painful experiences of life, where you push in one direction and the result goes off in another - a concept often not yet known by young carefree students. The effect of rotation on gravity currents can be particularly powerful in the presence of a boundary because a pressure gradient into the wall can easily be set up and drive a current which hugs the boundary 


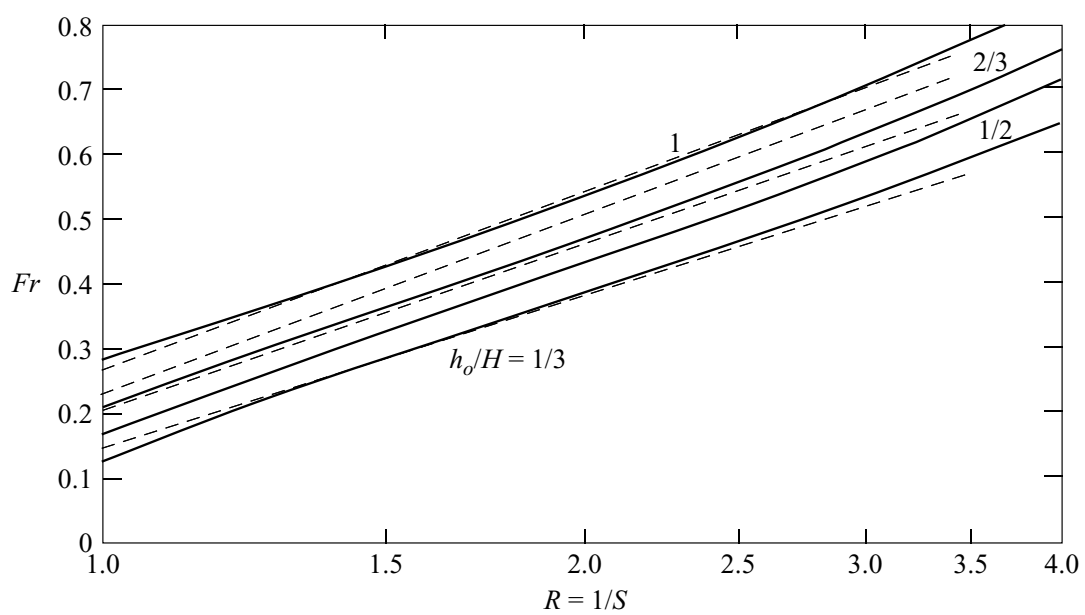

FIGURE 8. The Froude number $F r=u N / H$, as a function of the logarithm of the inverse stratification parameter $S$ for a high-Reynolds-number gravity current propagating with velocity $u$ beneath a stratified ambient of depth $H$ and buoyancy frequency $N$ for different initial depth ratios. The solid curves are the theoretical evaluations of Ungarish \& Huppert (2002) to be compared with the dashed curves which are the experimental results determined by Maxworthy et al. (2002).

(Griffiths \& Hopfinger 1983). These situations have been nicely reviewed in detail by Griffiths (1986).

An experimental investigation of axisymmetric gravity currents, where boundaries do not restrict the flow, was carried out at the large rotating system housed in the Coriolis laboratory at the Laboratoire des Ecoulements Geóphysiques et Industriales, Grenoble by Hallworth et al. (2001). The major new feature of the results was the attainment of a maximum radius of propagation, $r_{\max }$, attained in about one third of a period of revolution. The observed result had been predicted earlier by a theoretical investigation of the governing shallow-water equations by Ungarish \& Huppert (1998), who evaluated that $r_{\max } \approx 1.6 \mathscr{C}^{1 / 2}$, where $\mathscr{C}=\Omega_{o} /\left(h_{o} g^{\prime}\right)^{1 / 2}, \Omega$ is the rotation rate and the dense fluid, of reduced gravity $g^{\prime}$, was initially contained in a cylinder of radius $r_{o}$ and height $h_{o}$. Thereafter the motion in the fluid consisted of a contraction/relaxation/propagation of the current with a regular series of outwardly propagating pulses. The frequency of these pulses was observed to be slightly higher than inertial, independent of $h_{o}$ and $g^{\prime}$, with an amplitude of the order of magnitude of half the maximum radius, as illustrated in figure 9. Analysis of the subsequent motion $(\Omega t \gg 1)$ and the influence of a slope is discussed in Hunt et al. (2005), along with some interesting applications to atmospheric fronts.

Most volcanic eruptions are of sufficiently small scale that effects due to the rotation of the Earth are negligible. However, rotational effects may dominate the infrequent so-called super eruptions, for which the mass of magma erupted is estimated to exceed $10^{15} \mathrm{~kg}$, input to the base of the atmosphere at more than $10^{6} \mathrm{~m}^{3} \mathrm{~s}^{-1}$. The propagation of the consequential eruption cloud will be restrained by rotation, but may still reach diameters of up to $6000 \mathrm{~km}$ (Baines \& Sparks 2006). The last such super eruption was of Toba, in Sumatra, about 71000 years ago. When the next one will occur, to give volcanologists good data to compare their theoretical models with is not at present known. 

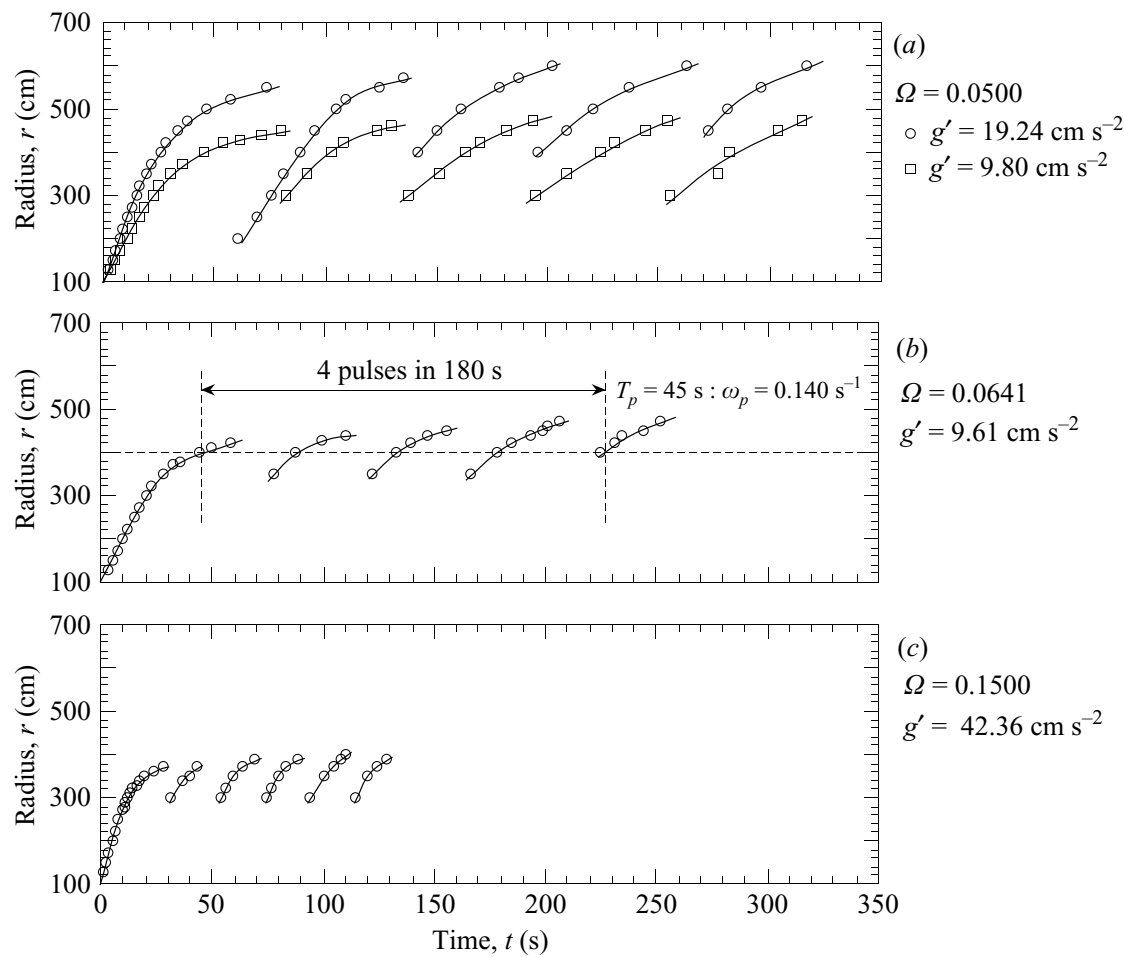

FIGURE 9. The radius of the leading edge of successive outward propagating fronts as a function of time for gravity currents intruding into a homogeneous rotating ambient at various rates of rotation $\Omega$ and reduced gravity $g^{\prime}$. The determination of the pulse frequency is shown as an example in $(b)$.

\section{Concentrated flows: mud, slurries and landslides}

As the concentration of particles increases, the assumption of low concentration employed in deriving (4.1) that each particle sediments with its independent Stokes freefall speed $V_{s}$ becomes less reliable. New effects must enter, and indeed, as the concentration becomes very high this situation relates more to mud flows than to the motion of suspended particles. Partly to investigate this problem, Hallworth \& Huppert (1998) carried out experiments on the instantaneous release of twodimensional heavy particle-driven gravity currents down a channel. Relatively low concentrations, with initial volume concentrations $\phi_{o}$ in the range $0<\phi_{o}<0.275$ were described well by the numerical solutions of (4.1) or the analytical box models associated with it, with the final runout length of the sedimented deposit increasing with $\phi_{o}$. Beyond this critical initial volume fraction of particles, the resulting dense current came to an abrupt halt at some point down the channel, depositing the bulk of its sediment load as a relatively thick layer of fairly constant depth, characterized by a pronounced steep snout. A very much thinner layer of sediment extended for some distance beyond the arrest point. This layer was deposited from the subsequent propagation of a slower moving low-concentration residual cloud and the final runout length of the sediment layer decreased with increasing $\phi_{o}$. This form of motion was observed for $0.275<\phi_{o}<0.45$, this upper limit being the approximate maximum for which Hallworth \& Huppert were able to produce a fluid-like initial volume. 
Amy et al. (2005) have suggested that this change in behaviour is related to a decrease in the Reynolds number of the flow, with initially high-Reynolds-number currents moving quickly from a slumping phase into the slowly moving viscous phase (and by-passing the inertia-buoyancy phase).

Over the last decade, motivated by its obvious natural applications, there has been considerable research to try to understand these high-concentration flows, exemplified by mud slides. Large mud flows can occur either in mountainous regions after intense rains and produce interesting alluvial fans or on submarine continental slopes, where they contribute to geomorphological evolution. There is hence considerable interest in both the geological and engineering community in such flows. Unfortunately, in contrast to all the work so far described in this article, for which it has been assumed that the constitutive relationship is Newtonian, it is quite definite that mud and high-concentration slurries are non-Newtonian. But how are they to be described? A plethora of rheological models have been proposed - power law, viscoplastic, Bingham plastic, Herschel-Bulkley, etc. Because of these different conceptual models, it is difficult to carry out systematic experiments which either test or verify any of these flows. Each person understandably views the possible contributions to the broad spectrum of science differently, but I find it unsatisfying working in areas where the fundamentals are on such shaky ground. Nevertheless, there has been considerable activity in this area, which might be broadly and briefly summarized as follows.

The main new effect that enters is the possibility of a critical yield stress, $\tau_{o}$, below which there is no shear possible in the flow. (There are similarities here to some of the models for non-Newtonian lava flows, nicely reviewed in Griffiths 2000.) This leads to a variety of features which are unknown in Newtonian fluids, even in quite simple situations. For example, a uniform layer of fluid mud can remain stationary on an incline, if the depth of the mud or the slope of the incline is sufficiently small. When motion does occur it is generally investigated under two approximations: either the flow is so slow that inertia is neglected (the more usual case); or so fast that a boundary-layer approximation is appropriate. A variety of different initial configurations have been solved for the motion of the mud and its final shape on a horizontal surface, down an inclined plane and over a gently sloped conical surface, representing a shallow basin or hill (Mei, Liu \& Yuhi 2001; Yuhi \& Mei 2004). Much further work in this area needs to be done before a good scientific understanding has been obtained and the results can be applied to the all-important area of hazard assessment, with the potential to save hundreds of thousands of lives and millions of wasted dollars.

\section{Granular flows}

The motion of granular media plays an important role in areas as diverse as agriculture, earth sciences and the pharmaceutical industry. The flow from a grain hopper for different shapes of the granular contents and the destructive collapse of such a hopper during flow are examples in industry on which millions of pounds per year are expended. The flow of granular material is arousing enormous research interest at the moment, as exemplified by the interesting broad reviews of Jaeger, Nagel \& Behringer (1996), Kadanoff (1999) and de Gennes (1999). Partially motivated by this interest, Gert Lube, Steve Sparks, Mark Hallworth and I conducted numerous experiments in which various granular materials, initially contained in a vertical cylinder, were rapidly released onto a horizontal surface to spread out unhindered over it (Lube et al. 2004, 2005, 2006). These studies and one carried out independently 
by Lajeunesse, Mangeney-Castelnau \& Vilotte (2004) have initiated many further investigations by other groups, whose contributions will be summarized at the end of this section. While not quite a fluid-mechanical gravity current, these flows are driven by density differences and have concepts in common with fluid-mechanical gravity currents, as outlined below.

Previous work on granular flows has considered thin quasi-steady layers flowing down inclined planes above the natural angle of repose for the grains, which is around $30^{\circ}$ for most granular material. The experimental results (see for example Pouliquen \& Forterre 2002) and equations developed to describe the flow (see for example Savage 1998) both depend on the basal and intergranular friction, with the equations incorporating empirical rheology expressions. Our approach was quite different in that it concentrated on rapid time-dependent flows for which both basal and intergranular friction are relatively immaterial, as is the situation in most natural and industrial flows. Part of our aim was also to relate the experimentally measured quantities directly to the appropriate input parameters.

The main materials we used in our experiments were dry sand and salt, but, for comparison purposes, we also conducted experiments with couscous, rice and sugar, all of which have a quite different grain size and shape. Nevertheless we found no significant difference in the resulting spreading relationships. The horizontal plane used was either wood, a smooth surface of baize lying on wood, a smooth transparent Plexiglas plane, glass or a rough plane of sandpaper. The detailed spread of the granular material was also independent of these surfaces because a few grains are quickly deposited on the base and the remaining grains flow over them, as described in more detail below. However, there are two extremes: perfectly smooth and round balls would travel a very long way, partially by rolling; regular blocks would not propagate at all - just like a stable tower of building blocks.

For the axisymmetric experiments eight different cylinders were used, while two different sorts of two-dimensional channel experiments were conducted. In the first set of experiments in a channel, the set-up was symmetrical, with a column of initial width $2 d_{i}$ collapsing, symmetrically, in opposite directions along the channel. In the second, a column of width $d_{i}$ supported by the vertical wall at the end of the channel collapsed (unsymmetrically) along the channel in one direction only. Some experiments were recorded on a digital high-speed video at 500 frames per second and later analysed. After all motion had ceased, the profile of the resultant deposit was measured to find the final runout radius, $r_{\infty}$, or runout length, $d_{\infty}$, central cone height, $h_{\infty}$, and the steepest incline, $\alpha$, of the upper surface.

For both geometries the collapse exhibited two sequential flow regimes. In the first, and more important, regime the free fall of the released grains was followed by lateral spreading of the grains with a static layer beneath a layer flowing at constant shear. The internal interface separating these two layers propagated mainly vertically through the granular medium until it reached the free surface, whereupon all bulk motion ceased. The second flow regime consisted of thin granular avalanches at the upper free surface, which slightly rearranged the top of the deposit. The first regime, and thus the main motion of the grains, was determined by inertial forces with intergranular frictional forces only playing a role in the second, avalanching regime.

The external input parameters describing the experiments are the initial radius $r_{i}$, or width $d_{i}$, the initial height $h_{i}$, and the gravitational acceleration $g$. From the two input variables with dimensions of length, an equivalent set can be defined which is $r_{i}$ or $d_{i}$, and the non-dimensional aspect ratio, $a$, defined by either $h_{i} / r_{i}$ or $h_{i} / d_{i}$, which is the important non-dimensional parameter describing the flow. 


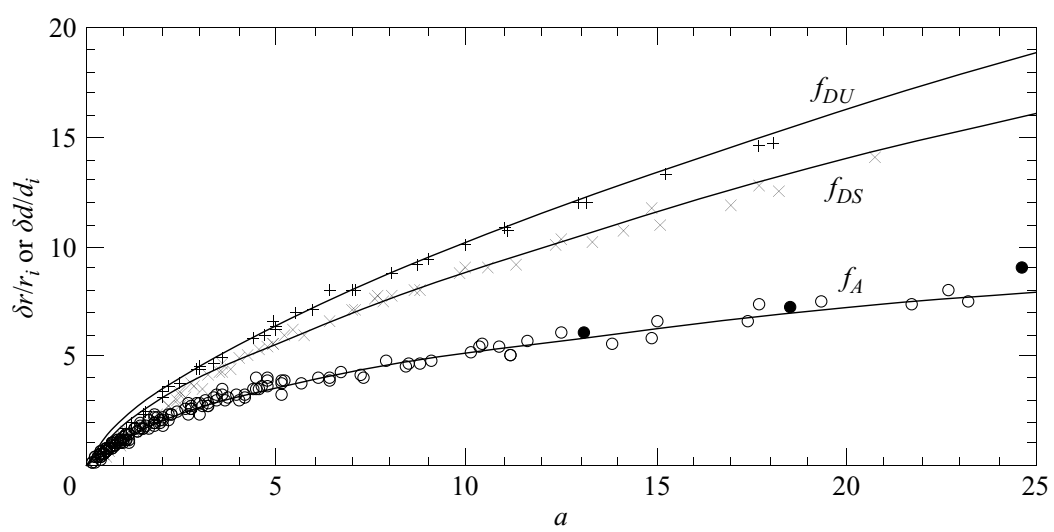

FIGURE 10. Non-dimensional final runout for the axisymmetric and two-dimensional collapses as functions of the aspect ratio. The curves are best fits and the symbols experimental data. The solid dots denote results from experiments with $H>0$.

For all values of $a$ there was a central undisturbed cone of material which did not partake in the motion, whose angle with the horizontal was close to $60^{\circ}$, corresponding to an aspect ratio of 1.7. This can be interpreted as an internal friction angle for the material. The value of $\alpha$ gradually decreased with $a$, to a value of $22 \pm 2^{\circ}$ for the axisymmetric and $27 \pm 2^{\circ}$ for the two-dimensional situation (Thompson \& Huppert 2006).

The quality of the observational data and the similarity of the results for the different materials suggest that, with the use of dimensional analysis, the data can be collapsed in a systematic way. Our ability to collapse all the data using only $r_{i}$ (or $d_{i}$ in the two-dimensional geometry), $a$ and $g$, and not any material properties, is a robust test for the assumption that no additional material property, such as the friction between individual grains, is needed to describe the motion.

The difference between the initial and final radius, $\delta r=r_{\infty}-r_{i}$, or $\delta d=d_{\infty}-d_{i}$ in the two-dimensional geometry, must be expressible as $\delta r=r_{i} f_{A}(a)$ or $\delta d=d_{i} f_{D}(a)$, where subscripts $A$ and $D$ refer to the axisymmetric and two-dimensional situations respectively. (This non-dimensionalization is formally correct in the two-dimensional geometry only if the width of the channel, $W$, considerably exceeds $h_{i}, d_{i}$ and $\delta d$.) Plots of $f_{A}$ and $f_{D}$ are given in figure 10. For $a<1.7$ (1.8 in the two-dimensional situation), because there is no motion of the inner region, the resultant expression must be independent of $r_{i}$ (or $d_{i}$ ), which requires that $f_{A}(a)$ and $f_{D}(a) \propto a$. From the data in figure 10 we determine that the constants of proportionality which best fit the data are 1.3,1.2 and 1.6 for the axisymmetric, two-dimensional symmetrical and unsymmetrical situations respectively, with the differences reflecting the different geometries. For $a>1.7$ (1.8) the best-fit power law to the data for all grains is given by $f(a)=1.6 a^{1 / 2}, f_{D S}(a)=1.9 a^{2 / 3}$ and $f_{D U}=2.2 a^{2 / 3}$, where the subscripts $S$ and $U$ refer to the (two-dimensional) symmetric and unsymmetric situations respectively. The difference between the power laws for the axisymmetric and two-dimensional situations reflects the different metric or geometry between the two situations.

The final height at the centre is similarly expressible as either $h_{\infty}=r_{i} \eta_{A}(a)$ or $d_{i} \eta_{D}(a)$. Figure 11 presents all the data for $h_{\infty} / r_{i}$ and $h_{\infty} / d_{i}$ as functions of $a$. For $a<1.7$ (1.2 in the two-dimensional situation), $h_{\infty}=h_{i}$, and so $\eta(a)=a$, as indicated by the data. In the axisymmetric geometry for $1.7<a \lesssim 10$ the best-fit power law to the data is given by $\eta_{A}(a)=0.88 a^{1 / 6}$. For $a \gtrsim 10$ there is a clear indication 


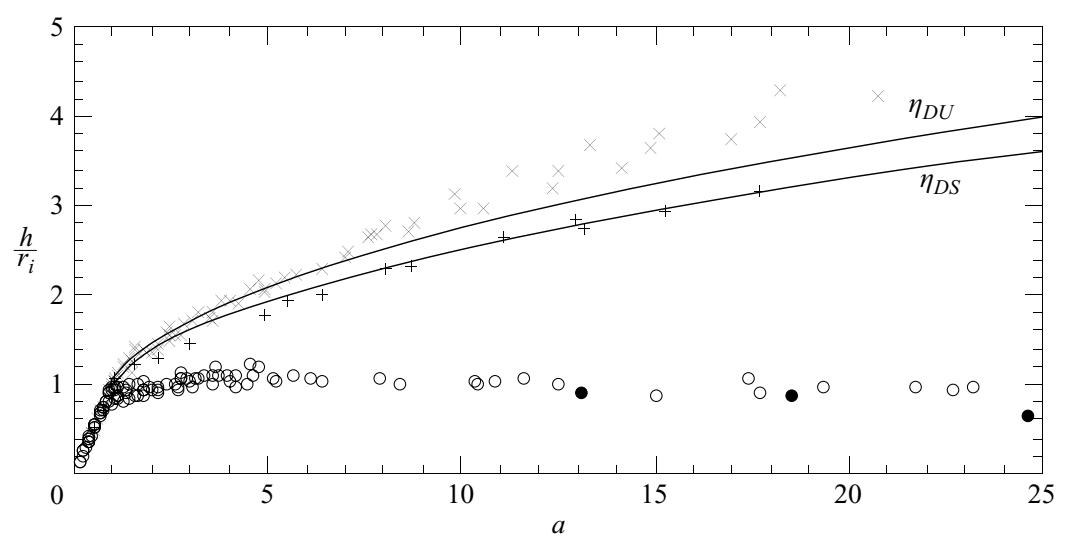

FIGURE 11. Non-dimensional maximum final height (at the centre of the pile for the axisymmetric and two-dimensional symmetric situations) as a function of the aspect ratio $a$.

that $h_{\infty}$ decreases with increasing $a$. This is in response to a wave that originates from the centre and removes material outwards. Unfortunately, the data are a little too scattered to be quantitatively analysed with confidence. In the two-dimensional geometry for $a>1.15, \eta_{D} \propto a^{2 / 5}$ with the constant of proportionality equal to 1.1 and 1.0 for the symmetric and asymmetric cases respectively.

As $a$ increases it becomes more and more difficult to release the grains instantaneously - too many of them initially stick to the walls as the cylinder is raised and then flow out. To counter this, Lube et al. (2004) carried out experiments with the granular media, of initial height $h_{i}$, raised a height $H$ before a constraining plug was released. Somewhat to our initial surprise the results for $r_{\infty}$ and $h_{\infty}$, considered now as functions of the extended aspect ratio $a_{H}=\left(H+h_{i}\right) / r_{i}$ dovetail well with the results for $H=0$, as indicated on figures 10 and 11 . The interpretation is that the final extent of the granular deposit and the final height at the centre are only dependent on the maximum height $h_{i}+H$ and the initial radius $r_{i}$, and not on most of the material below the maximum height. The deposit shape, however, must depend strongly on the initial volume of material, which determines the final volume of the deposit.

So far gravity $g$ has not entered our expressions because it is the only input parameter for which time is involved in its dimensions, and $\delta r$ (or $\delta d), h_{\infty} / r_{i}\left(\right.$ or $h_{\infty} / d_{i}$ ) are both independent of time. The value of $g$ will affect the total time for collapse, $t_{\infty}$, which is defined as the time between the initiation of the experiment and that at which the flow front stops propagating. The high-speed video allowed us to determine $t_{\infty}$ reasonably accurately. By dimensions, it must be of the form $t_{\infty}=\left(r_{i} / g\right)^{1 / 2} \psi_{A}(a)$ (or $\left(d_{i} / g\right)^{1 / 2} \psi_{D}(a)$ ) for some function $\psi(a)$. Plots of both $t_{\infty} /\left(r_{i} / g\right)^{1 / 2}$ and $t_{\infty} /\left(d_{i} / g\right)^{1 / 2}$ as functions of $a$ are presented in figure 12. As before, for small $a$, expressions for $\psi(a)$ independent of $r_{i}$ or $d_{i}$ require $\psi(a) \propto a^{1 / 2}$, which is consistent with the data. For large $a$ the best-fit power law through the data yields $\psi_{A}(a)=3.0 a^{1 / 2}$ and $\psi_{D}(a)=3.3 a^{1 / 2}$, which indicate that $t_{\infty \mathrm{A}}=3.0\left(h_{i} / g\right)^{1 / 2}$ and $t_{\infty D}=3.3\left(h_{i} / g\right)^{1 / 2}$, both still independent of $r_{i}$ or $d_{i}$ even for large $a$. Note that the time taken for a particle to fall a height $h_{i}$ under gravity is $\left(2 h_{i} / g\right)^{1 / 2}$, which is just less than a half of either of the $t_{\infty}$.

Further insight on the flow characteristics is obtained by an analysis of our kinematic data on the position of the flow front as a function of time. For all flows there was a primary acceleration phase during which the acceleration was 


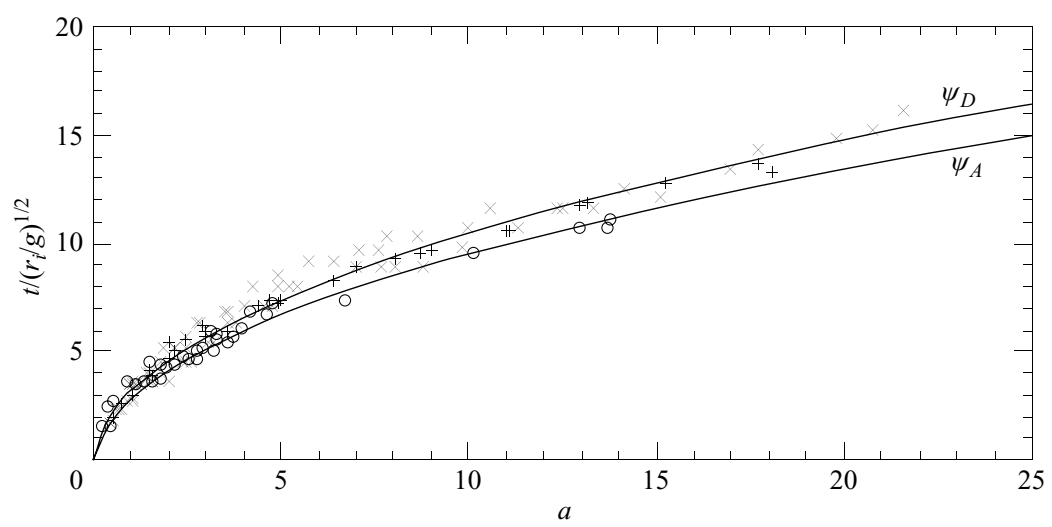

FIgURE 12. Non-dimensional time until the grain front ceased motion as a function of the aspect ratio.

approximately constant, with values between $0.25 \mathrm{~g}$ and $0.3 \mathrm{~g}$ for the axisymmetric geometry and $0.75 \mathrm{~g}$ for the two-dimensional situations. For $a<1.7$ (or 1.5 for the two-dimensional situations) this acceleration phase was followed by a phase of deceleration which came quite abruptly to a halt. For larger values of $a$ these two phases were separated by a phase of constant velocity of the flow front whose duration increased with increasing $a$. This phase of constant velocity is analogous to the evolution of a fluid gravity current spreading at high Reynolds number, which also goes through a stage of constant velocity (Huppert \& Simpson 1980). A fluid current then adjusts under a balance between buoyancy and inertial forces, spreading like $t^{1 / 2}$ (cf. (3.1)) - the same result as obtained for the granular collapses described here. This comparison, determined by using quantitative relationships to describe the motion of the granular medium that are closely analogous to those used to describe the motion of density currents of Newtonian liquids, further strengthens our argument that intergranular frictional effects play a negligible role until the abrupt halt commences.

Motivated by our axisymmetric experiments, Balmforth \& Kerswell (2005) carried out similar two-dimensional experiments. They focused on two materials with very different frictional properties, using mainly grit, with an overall mean size of approximately $1 \mathrm{~mm}$, and smooth glass beads of mean diameter $0.8 \mathrm{~mm}$. They conducted experiments both in a thin slot, where frictional effects at the walls would be dominant throughout the motion, and between widely spaced walls. Their experimental results for the latter are generally similar to ours, except they stress that they find a significant difference in the pre-multiplicative constants for the power-law relationship for the two grains used. This is not surprising, since smooth spherical balls would spread further, by rolling over both the horizontal surface and each other. They compare their experimental results with a shallow-water model, inputting suitable values for the friction coefficient, developed in Kerswell (2005), and find only qualitative agreement.

Obtaining the spreading relationships that the experiments have indicated remains a challenge for the future. A difficulty is that for at least half of the flow the assumptions of shallow-water theory, as applied by some to this problem, are false. An interesting counter example is offered by the imaginative study of Larrieu, Staron \& Hinch (2006) in this volume. They consider the first part of the collapse to consist of the free fall of the particles under gravity (and do not pretend that this can in any way be described 
by the shallow-water equations). They then apply the depth-averaged shallow-water equations for the remainder of the flow, gradually adding the grains to the layer at $r$ (or $x$ ) $=0$ with no horizontal momentum. The results are in good agreement with the experiments in the different geometries, though in detail this requires some adjustment of the two friction coefficients that appear in the equations. Larrieu et al. (2006) do not clarify how sensitive the results are to all of these assumptions. I am sure they intend to carry out further work along this line.

In the meantime, an additional clue to understanding the emplacement we have observed may come from an interpretation of the moving internal interface which separates static from flowing grains. It has been suggested that granular materials can be considered in two states (Jaeger et al. 1996): a static solid state, where intergranular forces at particle faces give the material strength; and a liquid state, exemplified by granular flows and fluidized beds, where the particles are in an agitated state and the system has negligible strength. The abrupt cessation of the motion of the granular flows that we observed can be likened to a phase change between two states (Huppert 1990). In accord with the characterization put forward in Jaeger et al. (1996), the change of state can be envisaged as a kinetic process analogous to solidification of a true liquid. In the case of a granular medium, we suggest that as the flow decelerates, the granular temperature falls below a threshold value and frictional interactions between particles become dominant and the granular material converts to a static solid (or deposit) at the moving internal interface.

This analogy can be pursued analytically by considering solidification at low values of the Stefan number, $S=L /\left(c_{p} \Delta T\right)$, where $L$ is the latent heat, $c_{p}$ the specific heat and $\Delta T$ the temperature fall needed for the fluid to solidify. Latent heat effects at the moving solidification interface are essential for the fluid to solidify, just as frictional effects are essential for the flow to cease. However, in the limit of low Stefan number, the time for solidification is independent of the explicit value of the latent heat, just as, we suggest, the time for motion to cease is independent of the friction.

An alternative, numerical approach to the problem would be to employ individual grain-following computations, which involve as many as $10^{8}$ grains. However, these have generally produced results which are heavily dependent upon intergranular friction and the exact shape of the grains (Zenit 2005; Staron \& Hinch 2006). Nevertheless, it might be interesting to pursue such an approach.

\section{Conclusions}

Since the propagation and evolution of gravity currents was first quantitatively analysed more than sixty years ago, a lot has been determined. The rates of propagation under many different situations have been evaluated, as described in the various sections of this article. The subject area has seen considerable active research over the last thirty years or so, primarily due to the influence of the giant names in the subject, including von Kármán (1940), Benjamin (1968) and Simpson (1997). Part of the drive for the research has come from the natural applications to flows in the oceans, atmosphere, and Earth's interior (Huppert 1986; Kerr \& Lister 1987) as well as to many engineering and industrial problems.

Many colleagues have helped me to understand the motion of gravity currents. I am grateful to each of them for their patience and wisdom. G. I. Barenblatt, M. A. Hallworth, R. C. Kerr, R. Kerswell, P. F. Linden, G. Lube, T. Maxworthy, A. Slim, R.S. J. Sparks, M. Ungarish and M. G. Worster kindly read an earlier version of 
this manuscript and provided useful reviews with suggestions for improvements. My research is supported by a Royal Society Wolfson Merit Award.

\section{REFERENCES}

Acton, J. M., Huppert, H. E. \& Worster, M. G. 2001 Two-dimensional viscous gravity currents flowing over a deep porous medium. J. Fluid Mech. 440, 359-380.

Amy, L. A., Hogg, A. J., Peakall, J. \& Talling, P. J. 2005 Abrupt transitions in gravity currents. J. Geophys. Res. 110, F03001, 1-19.

Baines, P. G. \& Sparks, R. S. J. 2006 Dynamics of giant volcanic ash clouds from supervolcano eruptions. Nature (to be submitted).

Balmforth, N. J. \& Kerswell, R. R. 2005 Granular collapses in two dimensions. J. Fluid Mech. 538, 399-428.

Balmforth, N. J., Burbidge, A. S. \& Craster, R. V. 2001 Shallow lava theory. In Geomorphological Fluid Mechanics (ed. N. Balmforth \& A. Provencale). pp. 164-187. Springer.

Balmforth, N. J., Craster, R. V. \& Sassi, R. 2004 Dynamics of cooling viscoplastic domes. J. Fluid Mech. 499, 149-182.

Barenblatt, G. I. 1978 Dynamics of turbulent spots and intrusions in a stably stratified fluid. Izvestiya 14, 139-145.

Barenblatt, G. I. 1996 Scaling, Self-similarity and Intermediate Asymptotics. Cambridge University Press.

Benjamin, T. B. 1968 Gravity currents and related phenomena. J. Fluid Mech. 31, 209-248.

Bickle, M., Chadwick, A., Huppert, H. E., Hallworth, M. A. \& Lyle, S. 2006 Analytical modelling of the carbon-dioxide plume at Sleipner: implications for underground carbon storage. Earth Planet Sci. Lett. (to be submitted).

Bonnecaze, R. T., Hallworth, M. A., Huppert, H. E. \& Lister, J. R. 1995 Axisymmetric particledriven gravity currents. J. Fluid Mech. 294, 93-125.

Bonnecaze, R. T., Huppert, H. E. \& Lister, J. R. 1993 Particle-driven gravity currents. J. Fluid Mech. 250, 339-369.

Chen, J. C. 1980 Studies on gravitational spreading currents. PhD thesis, California Institute of Technology.

Dalziel, S. B. \& Linden, P. F. 1997 Application of a general purpose CFD package to RayleighTaylor instability. In Proc. 6th Intl Workshop on Physics of Compressible Turbulent Mixing (ed. G. Jourdan \& L. Houas).

Didden, N. \& Maxworthy, T. 1982 The viscous spreading of plane and axisymmetric gravity currents. J. Fluid Mech. 121, 27-42.

FINK, J. H. \& GRIFFITHS, R. W. 1990 Radial spreading of viscous gravity currents in solidifying crust. J. Fluid Mech. 221, 485-510.

FInK, J. H. \& GRIFFITHS, R. W. 1998 Morphology, eruption rates, and rheology of lava domes; insights from laboratory models. J. Geophys. Rev. 103, 527-548.

DE Gennes, P. G. 1999 Granular matter: a tentative view. Rev. Mod. Phys 71, 374-382.

Griffiths, R. W. 1986 Gravity currents in rotating systems. Annu. Rev. Fluid Mech. 18, 59-89.

Griffiths, R. W. 2000 The dynamics of lava flows. Annu. Rev. Fluid Mech. 32, 477-518.

Griffiths, R. W. \& Hopfinger, E. 1983 Gravity currents moving along a lateral boundary in a rotating fluid. J. Fluid Mech. 134, 357-399.

Hacker, J., Linden, P. F. \& Dalziel, S. B. 1996 Mixing in lock-release gravity currents. Dyn. Atmos. Oceans 24, 183-195.

Hallworth, M. A. \& Huppert, H. E. 1998 Abrupt transitions in high-concentration, particle-driven gravity currents. Phys. Fluids 10, 1083-1087.

Hallworth, M. A., HogG, A. J. \& Huppert, H. E. 1998 Effects of external flow on compositional and particle gravity currents. J. Fluid Mech. 359, 109-142.

Hallworth, M. A., Huppert, H. E. \& Ungarish, M. 2001 Axisymmetric gravity currents in a rotating system: experimental and numerical investigations. J. Fluid Mech. 447, 1-29.

Hallworth, M. A., Huppert, H. E. \& Ungarish, M. 2003 On inwardly propagating axisymmetric gravity currents. J. Fluid Mech. 494, 255-274. 
Hallworth, M. A., Phillips, J., Huppert, H. E. \& Sparks, R. S. J. 1993 Entrainment in turbulent gravity currents. Nature 362, 829-831.

Hallworth, M. A., Phillips, J., Huppert, H. E. \& Sparks, R. S. J. 1996 Entrainment into two-dimensional and axisymmetric turbulent gravity currents. J. Fluid Mech. 308, 289-312.

Hallworth, M. A., Hogg, A. J. \& Huppert, H. E. 1998 Effects of external flow on compositional and particle gravity currents. J.Fluid Mech. 359, 109-142.

Harris, T. C., HogG, A. J. \& Huppert, H. E. 2001 A mathematical framework for the analysis of particle-driven gravity currents. Proc. R. Soc. Lond. A 457, 1241-1272.

Harris, T. C., Hogg, A. J. \& Huppert, H. E. 2002 Polydisperse particle-driven gravity currents. J. Fluid Mech. 472, 333-372.

Härtel, C., Meiburg, E. \& Necker, F. 2000 Analysis and direct numerical simulation of the flow at a gravity current head. Part 1 . Flow topology and front speed for slip and no-slip boundaries. J. Fluid Mech. 418, 189-212.

HogG, A. J. \& Huppert, H. E. 2001 a Two-dimensional and axisymmetric models for compositional and particle-driven gravity currents in uniform ambient flows. In Sediment Transport and Deposition by Particulate Gravity Currents. Spec. Publs. Intl Assoc. Sediment, vol. 31, pp. 121-134.

HogG, A. J. \& Huppert, H. E. $2001 b$ Spreading and deposition of particulate matter in uniform fluids. J. Hydraul. Res. 39, 505-518.

Hogg, A. J., Huppert, H. E. \& Hallworth, M. A. 2005 Constant flux currents in external mean flows. J. Fluid Mech. 539, 349-385.

Hoult, D. P. 1972 Oil spreading on sea. Annu. Rev. Fluid Mech. 4, 341-368.

Hunt, J. C. R., Pacheco, J. R., Mahalov, A. \& Fernando, H. J. S. 2005 Effects of rotation and sloping terrain on the fronts of density currents. J. Fluid Mech. 537, 285-315.

HuPPERT, H. E. 1982a The propagation of two-dimensional and axisymmetric viscous gravity currents over a rigid horizontal surface. J. Fluid Mech. 121, 43-58.

HUPPERT, H. E. $1982 b$ The flow and instability of viscous gravity currents down a slope. Nature 300, 427-429.

Huppert, H. E. 1986 The intrusion of fluid mechanics into geology. J. Fluid Mech. 173, 557-594.

Huppert, H. E. 1990 The fluid mechanics of solidification. J. Fluid Mech. 212, 209-240.

Huppert, H. E. 1998 Quantitative modelling of granular suspension flows. Phil. Trans. R. Soc. Lond. 356, 2471-2496.

Huppert, H. E. 2000 Geological fluid mechanics. In Perspectives in Fluid Dynamics: A Collective Introduction to Current Research (ed. G. K. Batchelor, H. K. Moffatt \& M. G. Worster), pp. 447-506. Cambridge University Press.

Huppert, H. E. \& Simpson, J. E. 1980 The slumping of gravity currents. J. Fluid Mech. 99, 785-799.

Huppert, H. E. \& Woods, A. W. 1995 Gravity-driven flows in porous layers. J. Fluid Mech. 292, 55-69.

Huppert, H. E., Shepherd, J. B., Sigurdsson, H. \& Sparks, R. J. S. 1982 On lava dome growth, with applications to the 1979 lava extrusion of Soufriere, St. Vincent. J. Volcanol. Geotherm. Res. 14, 199-222.

Jaeger, H. M., Nagel, S. R. \& Behringer, R. P. 1996 Granular solids, liquids and gases. Rev. Mod. Phys. 68, 1259-1273.

JaUPaRT, C. 1991 Effects on compressibility on the flow of lava. Bull. Volcanol. 54, 1-9.

KADANOFF, L. P. 1999 Built upon sand: Theoretical ideas inspired by granular flows. Rev. Mod. Phys. 71 (1), 435-444.

Kerr, R. C. \& Lister, J. R. 1987 The spread of subducted lithospheric material along the midmantle boundary. Earth Planet. Sci. Lett. 85, 241-247.

Kerswell, R. R. 2005 Dam break with Coulomb friction: A model for granular slumping? Phys. Fluids 17, 057101, 1-16.

Lajeunesse, E., Mangeney-Castelnau, A. \& Vilotte, J. P. 2004 Spreading of a granular mass on a horizontal plane. Phys. Fluids 16, 2371-2381.

Larrieu, E., Staron, L. \& Hinch, E. J. 2006 Raining into shallow water as a description of the collapse of a column of grains. J. Fluid Mech. 554, 259-270.

Lube, G., Huppert, H. E., Sparks, R. S. J \& Hallworth, M. A. 2004 Axisymmetric collapses of granular columns. J. Fluid Mech. 508, 175-199.

Lube, G., Huppert, H. E., Sparks, R. S. J. \& Freundt, A. 2005 Collapses of two-dimensional granular columns. Phys. Rev. E (in press). 
Lube, G., Huppert, H. E., Sparks, R. S. J. \& Freundt, A. 2006 Static and flowing regions in granular column collapses down channels. Phys. Fluids (submitted).

Lyle, S., Huppert, H. E., Hallworth, M. A., Bickle, M. \& Chadwick, A. 2005 Axisymmetric gravity currents in a porous medium. J. Fluid Mech. 543, 293-302.

Martin, D. \& Nokes, R. 1988 Crystal settling in a vigorously convecting magma chamber. Nature 332, 534-536.

Maxworthy, T., Lielich J., Simpson, J. E. \& Meiburg, E. H. 2002 Propagation of a gravity current in a linearly stratified fluid. J. Fluid Mech. 453, 371-394.

McKenzie D., Ford, P. G., Liu, F. \& Pettengill, G. H. 1992 Pancake-like domes on Venus. J. Geophys. Res. 97 (E10), 15967-15976.

Mei, C. C., Liu, K.-F. \& Yuhi, M. 2001 Mud flows - slow and fast. Geomorphological Fluid Mechanics (ed. N. Balmforth \& A. Provencale), pp. 548-577. Springer.

Pouliquen, O. \& Forterre, Y. O. 2002 Friction law for dense granular flows: application to the motion of a mass down a rough inclined plane. J. Fluid Mech. 453, 133-151.

Pritchard, D., Woods, A. W. \& HogG, A. J. 2001 On the slow draining of a gravity current moving through a layered permeable medium. J. Fluid Mech. 444, $23-47$.

Rottman, J. W. \& Simpson, J. E. 1983 Gravity currents produced by instantaneous releases of a heavy fluid in a rectangular channel. J. Fluid Mech. 135, 95-110.

Savage, S. B. 1998 Analyses of slow high-concentration flows of granular materials. J. Fluid Mech. 377, 1-26.

Simpson, J. E. 1997 Gravity Currents in the Environment and the Laboratory. Cambridge University Press.

Simpson, J. E. \& Britter, R. E. 1980 A laboratory model of an atmospheric mesofront. $Q . J . R$. Met. Soc. 106, 485-500.

Slim, A. C. \& Huppert, H. E. 2004 Self-similar solutions of the axisymmetric shallow-water equations governing converging inviscid gravity currents. J. Fluid Mech. 506, 331-355.

Staron, L. \& Hinch, E. J. 2006 Study of the collapse of granular columns using 2D discrete grains simulation. J. Fluid Mech. (submitted).

Thomas, L. P., Marino, B. M. \& Linden, P. F. 1998 Gravity currents over porous substrate. J. Fluid Mech. 366, 239-258.

Thomas, L. P., Marino, B. M. \& Linden, P. F. 2004 Lock-release inertial gravity currents over a thick porous layer. J. Fluid Mech. 503, 299-319.

Thompson, E. L. \& Huppert, H. E. 2006 Granular column collapses: further experimental results. Phys. Fluids (submitted).

Turner, J. S. 1979 Buoyancy Effects in Fluids. Cambridge University Press.

UNGARISH, M. 2005 Intrusive gravity currents in a stratified ambient: shallow-water theory and numerical results. J. Fluid Mech. 535, 287-323.

Ungarish, M. \& Huppert, H. E. 1998 The effects of rotation on axisymmetric, particle-driven gravity currents. J. Fluid Mech. 362, 17-51.

Ungarish, M. \& HuPPERT, H. E. 2000 High-Reynolds-number gravity currents over a porous boundary: shallow-water solutions and box model approximations. J. Fluid Mech. 418, 1-23.

UNGARISH, M. \& HuPPERT, H. E. 2002 On gravity currents propagating at the base of a stratified ambient. J. Fluid Mech. 458, 283-301.

UNGARISH, M. \& HUPPERT, H. E. 2004 On gravity currents propagating at the base of a stratified ambient: effects of geometrical constraints and rotation. J. Fluid Mech. 521, 69-104.

UNGARISH, M. \& HuPPERT, H. E. 2006 Energy balances for propagating gravity currents: homogeneous and stratified ambients. J. Fluid Mech. (submitted).

Vella, D. \& Huppert, H. E. 2006 Gravity currents in a porous medium at an inclined plane. J. Fluid Mech. (submitted).

von KÁRmán, T. 1940 The engineer grapples with nonlinear problems. Bull. M. Math. Soc. 46, 615-683.

Whitham, G. B. 1974 Linear and Nonlinear Waves. Wiley.

Yuni, M. \& MeI, C. C. 2004 Slow spreading of fluid-mud over a conical surface. J. Fluid. Mech. 519, 337-358.

Zenit, R. 2005 Computer simulations of the collapse of a granular column. Phys. Fluids 17, 031703, $1-4$. 